\title{
Hamilton cycles in sparse locally connected graphs
}

van Aardt, Susan A.; Burger, Alewyn P.; Frick, Marietjie; Thomassen, Carsten; de Wet, Johan P.

Published in:

Discrete Applied Mathematics

Link to article, DOI:

10.1016/j.dam.2018.10.031

Publication date:

2019

Document Version

Peer reviewed version

Link back to DTU Orbit

Citation $(A P A)$ :

van Aardt, S. A., Burger, A. P., Frick, M., Thomassen, C., \& de Wet, J. P. (2019). Hamilton cycles in sparse locally connected graphs. Discrete Applied Mathematics, 257. https://doi.org/10.1016/j.dam.2018.10.031

\section{General rights}

Copyright and moral rights for the publications made accessible in the public portal are retained by the authors and/or other copyright owners and it is a condition of accessing publications that users recognise and abide by the legal requirements associated with these rights.

- Users may download and print one copy of any publication from the public portal for the purpose of private study or research.

- You may not further distribute the material or use it for any profit-making activity or commercial gain

- You may freely distribute the URL identifying the publication in the public portal

If you believe that this document breaches copyright please contact us providing details, and we will remove access to the work immediately and investigate your claim. 


\title{
Hamilton cycles in sparse locally connected graphs
}

\author{
Susan A. van Aardt ${ }^{\mathrm{a}}$, Alewyn P. Burger ${ }^{\mathrm{b}}$, Marietjie Frick ${ }^{\mathrm{c}}$, Carsten Thomassen ${ }^{\mathrm{d}}$, \\ Johan P. de Wet ${ }^{\mathrm{c}, \mathrm{e}, *}$

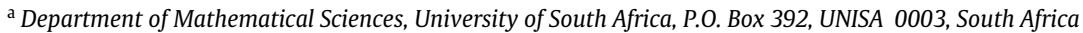 \\ ${ }^{\mathrm{b}}$ Department of Logistics, University of Stellenbosch, Private Bag X1, Matieland, 7602, South Africa \\ ${ }^{c}$ Department of Mathematics and Applied Mathematics, University of Pretoria, Private Bag X20, Hatfield 0028, South Africa \\ ${ }^{\mathrm{d}}$ Department of Applied Mathematics and Computer Science, Technical University of Denmark, DK-2800 Lyngby, Denmark \\ e DST-NRF Centre of Excellence in Mathematical and Statistical Sciences (CoE-MaSS), South Africa
}

\section{A R T I C L E I N F O}

\section{Article history:}

Received 20 December 2016

Received in revised form 5 January 2018

Accepted 20 October 2018

Available online $\mathrm{xxxx}$

\section{Keywords:}

Locally connected

Hamiltonian

NP-complete

Polynomial time algorithm

\begin{abstract}
A B S T R A C T
A graph $G$ is locally connected if for every $v \in V(G)$ the open neighbourhood $N(v)$ of $v$ is nonempty and induces a connected graph in $G$. We characterize locally connected graphs of order $n$ with less than $2 n$ edges and show that for any natural number $k$ the Hamilton Cycle Problem for locally connected graphs of order $n$ with $m$ edges is polynomially solvable if $m \leq 2 n+k \log _{2} n$, but NP-complete if $m=2 n+\left\lfloor n^{1 / k}\right\rfloor$.
\end{abstract}

(C) 2018 Elsevier B.V. All rights reserved.

\section{Introduction and background}

Let $G$ be a graph with vertex set $V(G)$ and edge set $E(G)$. The cardinalities of $V(G)$ and $E(G)$ are called the order and size of $G$ and denoted by $n(G)$ and $m(G)$, respectively. The minimum and maximum degrees of $G$ are denoted by $\delta(G)$ and $\Delta(G)$, respectively. When the context is clear we omit the $G$ and simply write $n, m, \Delta$ etc. For undefined concepts and notation we refer the reader to [1].

A graph $G$ is locally connected $(L C)$ if for every $v \in V(G)$ the open neighbourhood $N(v)$ of $v$ is nonempty and induces a connected graph. The concept of a locally connected graph was introduced by Chartrand and Pippert [2]. One of the first results proved on the topic is the following.

Theorem 1.1 ([2]). If $G$ is a connected LC graph of order at least 3 with $\Delta \leq 4$, then $G$ is either hamiltonian or isomorphic to the complete tripartite graph $K_{1,1,3}$.

Global cycle properties of locally connected graphs with $\Delta=5$ were investigated by Kikust [8], Clark [3], Hendry [5] and Gordon, Orlovich, Pots and Strusevich [4]. Hendry [6] defined a graph $G$ to be fully cycle extendable if every vertex of $G$ lies on a 3-cycle and for every nonhamiltonian cycle $C$ in $G$ there is a cycle $C^{*}$ in $G$ that contains all the vertices of $C$ plus a single new vertex. The combined results of the mentioned authors yield the following.

Theorem $1.2([3-5,8])$. If $G$ is a connected $L C$ graph with $\Delta=5$ and $\delta \geq 3$, then $G$ is fully cycle extendable. However, there exist infinitely many nonhamiltonian connected LC graphs with $\Delta=5$ and $\delta=2$.

\footnotetext{
* Corresponding author at: Department of Mathematics and Applied Mathematics, University of Pretoria, Private Bag X20, Hatfield 0028, South Africa. E-mail addresses: vaardsa@unisa.ac.za (S.A.van Aardt), apburger@sun.ac.za (A.P. Burger), marietjie.frick@up.ac.za (M. Frick), ctho@dtu.dk (C. Thomassen), johan.dewet@up.ac.za (J.P. de Wet).
} 


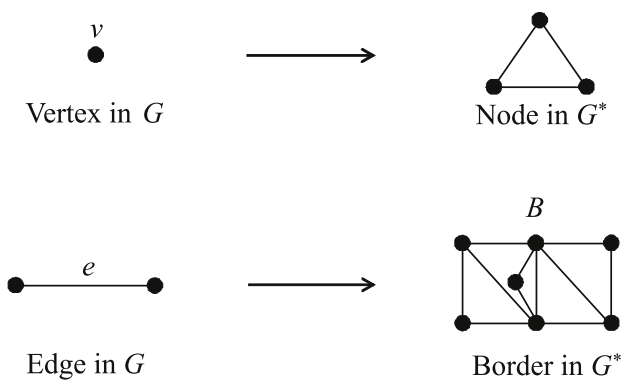

Fig. 1. The translation of vertices and edges in $G$ to nodes and borders in $G^{*}$.

It is well-known that the Hamilton Cycle Problem (the problem of deciding whether a given graph is hamiltonian) is NP-complete.

Henceforth we abbreviate Hamilton Cycle Problem to HCP. Theorem 1.1 shows that the HCP for LC graphs with $\Delta \leq 4$ is fully solved. Gordon et al. [4] proved that it is NP-complete for LC graphs with $\Delta=7$. Irzhavski [7] improved on this result by proving the following:

Theorem 1.3 ([7]). The HCP for LC graphs with minimum degree 2 and maximum degree 5 is NP-complete. Moreover, for any two integers $d$ and $D$ such that $D \geq 6$ and $2 \leq d \leq D$, the HCP for locally connected graphs with minimum degree $d$ and maximum degree $D$ is NP-complete.

We would like to thank the referee who made us aware of Irzhavski's theorem.

If $\mathcal{R}$ is a finite set of natural numbers, we say a graph $G$ is $\mathcal{R}$-regular if the degrees of the vertices in $G$ are all elements of $\mathcal{R}$. In Section 2 we state a corollary of Irzhavski's theorem in terms of $\mathcal{R}$-regular graphs. We also use his technique to prove one of our main results in Section 4. Since Irzhavski's paper [7] has only been published in Russian, we include the main steps of the proof for easy access.

Theorem 1.1 prompted us to investigate the HCP for LC graphs with average degree at most 4, i.e., graphs with $m \leq 2 n$. In Section 3 we characterize LC graphs with $m<2 n$. In Section 4 we show that for any natural number $k$ the HCP is NP-complete for LC graphs with $m=2 n+\left\lfloor n^{1 / k}\right\rfloor$, but polynomially solvable for LC graphs with $m \leq 2 n+k \log n$.

\section{Hamilton cycles in R-regular LC graphs}

Picouleau [9] proved the following result regarding the complexity of the HCP for $r$-regular graphs.

Theorem 2.1 ([9]). For any fixed integer $r \geq 3$ the HCP for $r$-regular graphs is NP-complete.

The problem of the complexity of the HCP for $\mathcal{R}$-regular LC graphs is settled by Theorem 1.2 and the following result, which is a corollary of Theorem 1.3, proved by Irzhavski [7].

Theorem 2.2 ([7]). The HCP for $\mathcal{R}$-regular LC graphs is NP-complete if $\mathcal{R}=\{2,5\}$ or $\mathcal{R}$ is any set of natural numbers with $\max \mathcal{R} \geq 6$.

Proof. It is sufficient to prove the cases $\mathcal{R}=\{2,5\}$ and $\mathcal{R}=\{r\}, r \geq 6$.

Consider any cubic graph $G$. By Theorem 2.1 it is sufficient to show that $G$ can be transformed in polynomial time to an $\mathcal{R}$-regular LC graph $G^{*}$ such that $G^{*}$ is hamiltonian if and only if $G$ is hamiltonian. We briefly explain Irzhavski's transformation for the cases $\mathcal{R}=\{2,5\}$ and $\mathcal{R}=\{6\}$.

For the case $\mathcal{R}=\{2,5\}$, the graph $G^{*}$ is constructed by replacing each vertex in $G$ with a triangle (henceforth called a node of $G^{*}$ ) and each edge in $G$ with a border isomorphic to the graph $B$ shown in Fig. 1, so that neighbouring nodes of $G^{*}$ are joined by borders as shown in Fig. 2. It is easily seen that $G^{*}$ is $\mathrm{LC}$ and $\{2,5\}$-regular.

For a vertex $v_{1}$ in $G$ with $N\left(v_{1}\right)=\left\{u_{1}, u_{2}, u_{3}\right\}$, let $V_{1}$ and $U_{i}, i=1,2,3$ be the nodes in $G^{*}$ corresponding to $v_{1}$ and $u_{i}$, $i=1,2,3$, respectively and let $B_{i}$ be the borders in $G^{*}$ corresponding to the edges $v_{1} u_{i}, i=1,2$, 3 . Let the vertices $a, b, c, d, x$ be as shown in Fig. 3.

Without loss of generality, suppose $G$ has a Hamilton cycle $C$ that contains the path $u_{1} v_{1} u_{2}$. Then $C$ also contains the path $v_{2} u_{3} v_{3}$, where $N\left(u_{3}\right)=\left\{v_{1}, v_{2}, v_{3}\right\}$. Those two paths correspond, for example, to the two paths in $G^{*}$ indicated by thick lines in Fig. 3. Hence, for any Hamilton cycle in $G$, there is a corresponding Hamilton cycle in $G^{*}$.

Now suppose $G^{*}$ has a Hamilton cycle $C^{*}$. If $C^{*}$ contains the pair $a d, b c$ of "parallel edges", then $C^{*}$ obviously contains the path $a d x c b$. It follows that $C^{*}$ contains at most one pair of parallel edges incident with any given node of $G^{*}$.

Now suppose $C^{*}$ contains no pair of parallel edges incident with $V_{1}$. Since $c x d$ is a subpath of $C^{*}$, at most one of the edges $\{a d, b d, b c\}$ of $B_{3}$ lies on $C^{*}$. The same is true for the corresponding edges of the borders $B_{1}$ and $B_{2}$. The vertices of $V_{1}$ therefore 
Vertices and edges in $G$

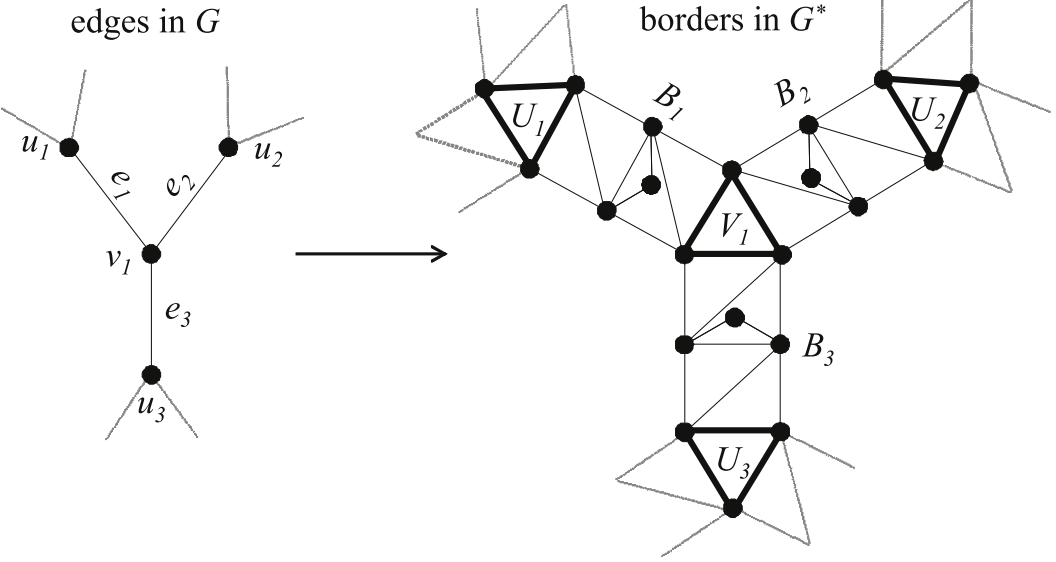

Fig. 2. A node in a $\{2,5\}$-regular LC graph $G^{*}$ connected with borders to three other nodes in $G^{*}$.

\section{Subpaths of a}

Hamilton cycle in $G$

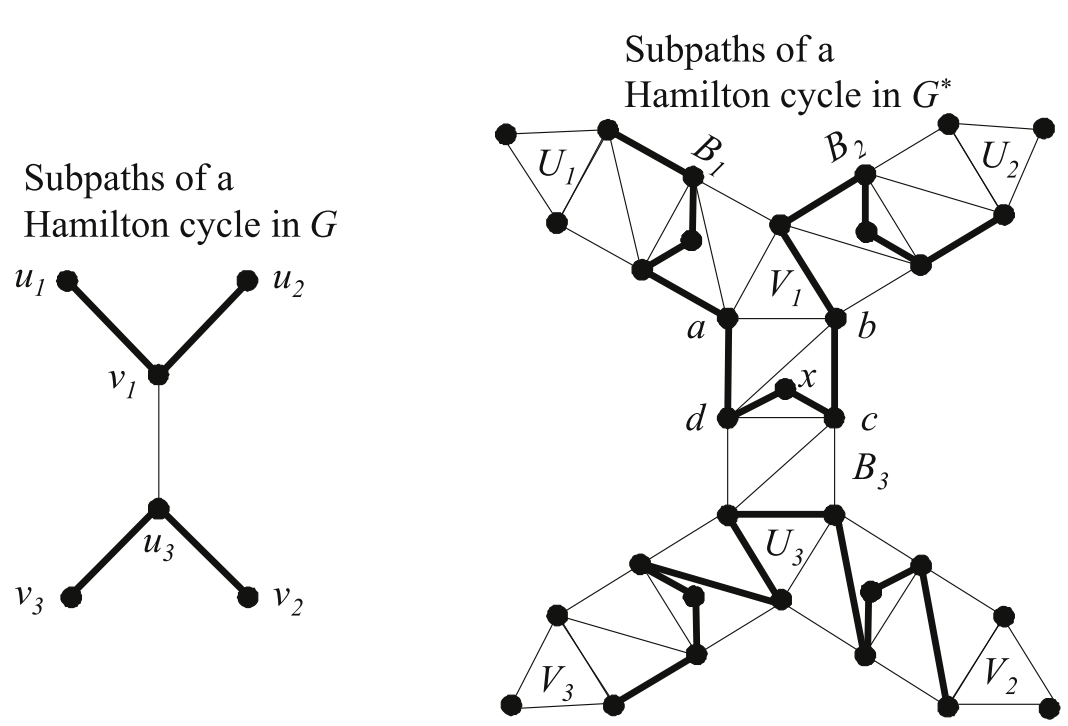

Nodes and borders in $G^{*}$

Fig. 3. Illustrating how a Hamilton cycle in $G$ translates to a Hamilton cycle in $G^{*}$.

lie on a $P_{3}$ in $C^{*}$, and in one of the borders, say $B_{1}$, the cycle $C^{*}$ has no edge of the form $a d, b c$ or $d b$ incident with $V_{1}$. But then $C^{*}$ contains the pair of parallel edges of $B_{1}$ incident with $U_{1}$. Thus $C^{*}$ translates to a Hamilton cycle in $G$. This proves the result for the case $\mathcal{R}=\{2,5\}$.

Fig. 4 illustrates how two nodes are joined by a border to effect the transformation in the case $\mathcal{R}=\{6\}$. (The sketch that appears in [7] has been redrawn so as to show clearly how the construction for the $\{2,5\}$-case has been modified to obtain a 6-regular LC graph.)

For $r \geq 7$ it is not difficult to obtain an $r$-regular LC graph with the desired properties by expanding the nodes and borders in the $\{2,5\}-$ construction.

\section{Characterization of the connected LC graphs with $m<2 n$}

We first prove the following.

Proposition 3.1. If $G$ is a connected $L C$ graph of order $n$ and size $m$, then $m \geq 2 n-3$.

Proof. The proof is by induction on $n$. The result is obviously true if $n \leq 3$. Now let $G$ be a connected LC graph of order $n \geq 4$ and size $m$. Let $x$ be a vertex of minimum degree in $G$. We may assume $d(x)=2$ or 3 . Let $H=G-x$.

If $d(x)=2$, then $H$ is obviously a connected LC graph of order $n-1$ and size $m-2$. Hence, by our induction hypothesis, $m-2 \geq 2(n-1)-3=2 n-5$, so then $m \geq 2 n-3$. 


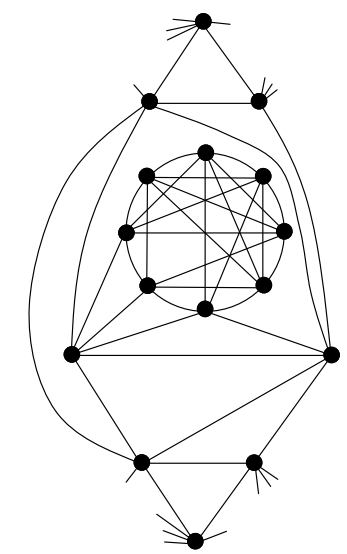

Fig. 4. A 6-regular LC construction.

If $d(x)=3$, there are two cases to consider.

Case 1. $G[N(x)] \cong K_{3}$ : In this case $H$ is a connected LC graph of size $m-3$ and hence, by our induction hypothesis, $m-3 \geq 2 n-5$, so $m \geq 2 n-2$.

Case 2. $G[N(x)]$ is a path $v_{1} v_{2} v_{3}$ : In this case the graph $H+v_{1} v_{3}$ is connected and LC and has size $m-2$. Hence, by our induction hypothesis, $m-2 \geq 2 n-5$, so $m \geq 2 n-3$.

Suppose that for $i=1,2$, the graph $G_{i}$ has a proper induced subgraph $H_{i}$ that is isomorphic to a given graph $H$. If we identify the subgraphs $H_{1}$ and $H_{2}$ while retaining all other edges present in $G_{1}$ and $G_{2}$, the resulting graph $G$ of order $n(G)=n\left(G_{1}\right)+n\left(G_{2}\right)-n(H)$ is said to be obtained from $G_{1}$ and $G_{2}$ by pasting $G_{1}$ and $G_{2}$ together on a copy of $H$. We observe the following.

Observation 3.2. Let $H$ be any graph with $\delta(H) \geq 1$ and suppose $G$ is a graph consisting of two LC graphs pasted together on a copy of $H$. Then $G$ is also $L C$.

If an LC graph $G$ consists of two LC graphs of order at least 3 each, pasted together on an edge (i.e., on a copy of $K_{2}$ ), we say that $G$ is a reducible LC graph. If $G$ is an LC graph that consists of more than one component, we also consider $G$ to be reducible, since each of its components is a connected LC graph. We define an irreducible LC graph $G$ as a graph that is not reducible.

A set of vertices $S$ in a connected graph $G$ is a vertex cut if $G-S$ is disconnected. If $\{x, y\}$ is a vertex cut in a connected LC graph $G$, then it is easily seen that $x y \in E(G)$. This implies the following.

Observation 3.3. If $G$ is an irreducible $L C$ graph of order $n$, then $G \in\left\{K_{2}, K_{3}\right\}$ if $n \leq 3$, and $G$ is 3-connected if $n \geq 4$.

In order to characterize the connected LC graphs with $m<2 n$ it suffices to characterize the irreducible ones, as the next result shows.

Proposition 3.4. If $G$ is a connected reducible LC graph with $m<2 n$, then $G$ is obtained by pasting irreducible LC graphs of size less than twice the order together along edges.

Proof. The proof is by induction on $n$. By assumption, $G$ is the union of two LC graphs $G_{1}, G_{2}$ having two vertices $x, y$ and the edge $x y$ in common. For $i=1,2$, let $n_{i}, m_{i}$ be the number of vertices and edges, respectively, in $G_{i}$. Proposition 3.1 implies that $m_{i} \geq 2 n_{i}-3$ for $i=1,2$. As $n=n_{1}+n_{2}-2$ and $m=m_{1}+m_{2}-1$, it follows that $m_{i}<2 n_{i}$ for $i=1,2$, and now the result follows by induction.

A vertex $u$ in a graph $G$ is called a universal vertex if $u$ is adjacent to every vertex in $G-u$. A graph is called a wheel if it consists of a cycle (called the rim of the wheel) plus a universal vertex (called the centre of the wheel). Fig. 5 depicts a wheel of order 9.

Let $W$ be a wheel with centre $w$ and $\operatorname{rim} w_{1} w_{2} \ldots w_{t}$. The edges $w w_{i}, i=1, \ldots, t$ are called the spokes of $W$. A wheel of order $n$ may be extended to a wheel of order $n+1$ by inserting a spoke, i.e., by subdividing an edge on the rim of $W$ and joining the inserted vertex to the centre of $W$. The reverse of this action is called eliminating a spoke.

If an edge on the rim of a wheel is deleted, the resulting graph is a fan. Fig. 6 depicts a fan of order 9. Thus a fan is a path plus a universal vertex. Let $F$ be a fan with central vertex $v$ and $\operatorname{rim} v_{1} \ldots v_{p}$. The edges $v v_{1}$ and $v v_{p}$ are the outer spokes of $F$, and $v v_{2}, \ldots, v v_{p-1}$ the inner spokes. A fan may be extended by inserting a spoke between two of its spokes, or by adding a 


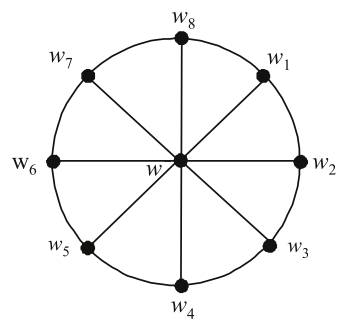

Fig. 5. A wheel of order 9 .

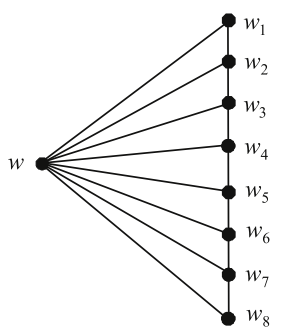

Fig. 6. A fan of order 9 .
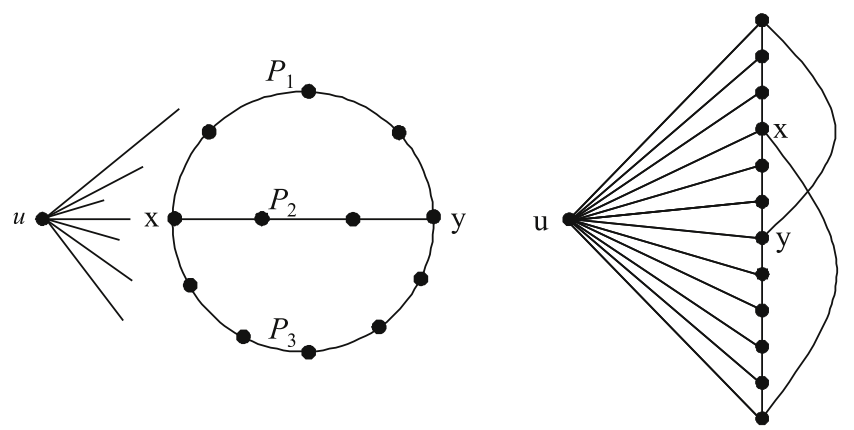

Fig. 7. Two depictions of an extended $\theta$-graph.

new vertex and joining it to the central vertex and an end vertex of the rim. A fan may be reduced by eliminating an internal spoke or deleting an end vertex of the rim (together with its two incident edges).

A $\theta$-graph is the union of three internally disjoint paths with shared end-vertices. An extended $\theta$-graph consists of a $\theta$ graph plus a universal vertex.

An extended $\theta$-graph may be viewed as two wheels $W$ and $Y$ pasted together on a fan $F$ such that the centre of $Y$ is pasted to the centre of $W$. If $F$ is a triangle (a fan with just two spokes) the result is a wheel plus an edge. An example of an extended $\theta$-graph is depicted in Fig. 7.

Note that a wheel of order 4 is a $K_{4}$, and any vertex in a $K_{4}$ may be regarded as its centre. Thus pasting a $K_{4}$ and any other wheel together on a triangle necessarily results in an extended $\theta$-graph.

If two wheels $W$ and $Y$ of order at least 5 each are pasted together on a triangle such that the centre of $Y$ is pasted to a vertex on the rim of $W$, the result is called a triangle bicycle.

If two wheels $W$ and $Y$ of order at least 5 each are pasted along a diamond (that is, a $K_{4}$ minus an edge) such that the centre of $Y$ is pasted to a vertex on the rim of $W$, the result is called a diamond bicycle.

For example, let $W$ be a wheel with centre $w$ and $\operatorname{rim} w_{1} w_{2} \ldots w_{8} w_{1}$ and let $Y$ be a wheel with centre $y$ and rim $y_{1} y_{2} \ldots y_{5} y_{1}$. Fig. 8 depicts a triangle bicycle, obtained from $W$ and $Y$ by pasting the triangle $Y\left[\left\{y, y_{1}, y_{2}\right\}\right]$ to the triangle $W\left[\left\{w_{1}, w_{2}, w\right\}\right]$. Fig. 9 depicts a diamond bicycle, obtained from $W$ and $Y$ by pasting the diamond $Y\left[\left\{y, y_{2}, y_{1}, y_{3}\right\}\right]$ to the diamond $W\left[\left\{w_{2}, w, w_{3}, w_{1}\right\}\right]$.

Theorem 3.5. $G$ is an irreducible $L C$ graph with $m(G) \leq 2 n(G)-1$ if and only if $G \in \mathcal{T}_{1} \cup \mathcal{T}_{2} \cup \mathcal{T}_{3}$, where

- $\mathcal{T}_{3}=\left\{K_{2}, K_{3}\right\}$.

- $\mathcal{T}_{2}$ consists of all the wheels.

- $\mathcal{T}_{1}$ consists of the extended $\theta$-graphs, the diamond bicycles and the triangle bicycles. 


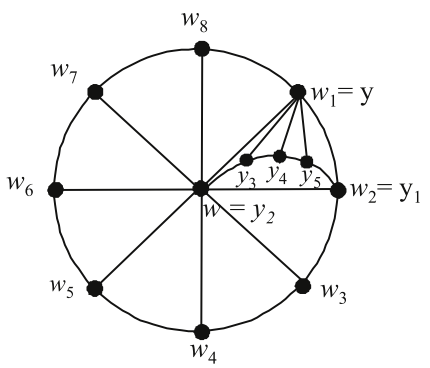

Fig. 8. A triangle bicycle.

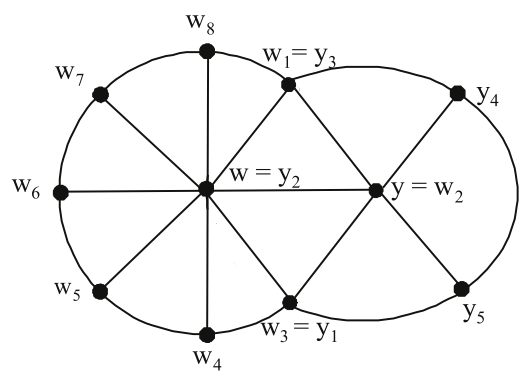

Fig. 9. A diamond bicycle.

Proof. Adding a universal vertex to any connected graph obviously results in an LC graph. This, together with Observation 3.2, implies that every graph in $\mathcal{T}_{1} \cup \mathcal{T}_{2} \cup \mathcal{T}_{3}$ is LC. It is easily seen that every graph in $\mathcal{T}_{1} \cup \mathcal{T}_{2}$ is 3-connected and that every graph in $\mathcal{T}_{i}$ satisfies $m=2 n-i, i=1,2$, 3. Thus, if $G \in \mathcal{T}_{1} \cup \mathcal{T}_{2} \cup \mathcal{T}_{3}$, then $G$ is an irreducible LC graph with $m(G) \leq 2 n(G)-1$.

To prove the converse, suppose $G$ is an irreducible LC graph with $m(G) \leq 2 n(G)-1$. We prove by induction on the order that $G \in \mathcal{T}_{1} \cup \mathcal{T}_{2} \cup \mathcal{T}_{3}$.

If $n(G) \leq 3$, then $G \in \mathcal{T}_{3}$, so the basis step is proved.

Now suppose $n(G) \geq 4$. Then $G$ is 3-connected by Observation 3.3. Since $m(G)<2 n(G)$, it therefore follows that $G$ has a vertex, say $x$, of degree 3. Let $H=G-x$. Since $G$ is locally connected, $G[N(x)]$ is isomorphic to either $K_{3}$ or $P_{3}$.

We now consider three cases, depending on the relative size of $G$. In each case we use the fact that if $G[N(x)]$ is a $K_{3}$, then $H$ is an irreducible LC graph, and if $G[N(x)]$ is a path $v_{1} v_{2} v_{3}$, then $H+v_{1} v_{3}$ is an irreducible LC graph. Note that in the latter case, $G$ can be retrieved from $H$ by subdividing the edge $v_{1} v_{3}$ with a vertex $x$ and then adding the edge $x v_{2}$.

Case 1. $m(G)=2 n(G)-3$ :

If $G[N(x)]$ is a $K_{3}$, then $m(H)=m(G)-3=2 n(H)-4$, contradicting Proposition 3.1. Hence we may assume that $G[N(x)]$ is a path $v_{1} v_{2} v_{3}$. Then $m\left(H+v_{1} v_{3}\right)=2 n(H)-3$. It therefore follows from our induction hypothesis that $H+v_{1} v_{3} \in \mathcal{T}_{3}$, so $H+v_{1} v_{3}$ is a $K_{3}$. But then $G$ is a diamond, contradicting that $G$ is irreducible. Hence $K_{2}$ and $K_{3}$ are the only irreducible LC graphs with $m=2 n-3$.

Case 2. $m(G)=2 n(G)-2$ :

If $G[N(x)]$ is a $K_{3}$, then $m(H)=2 n(H)-3$, so then it follows from our induction hypothesis that $H \in \mathcal{T}_{3}$ and hence $H \cong K_{3}$. Then $G \cong K_{4}$, so $G \in \mathcal{T}_{2}$.

Now suppose $G[N(x)]$ is a path $v_{1} v_{2} v_{3}$. Then $m\left(H+v_{1} v_{3}\right)=2 n\left(H+v_{1} v_{3}\right)-2$. Hence, by our induction hypothesis, $H+v_{1} v_{3}$ is a wheel $W$. Since the set $\left\{v_{1}, v_{2}, v_{3}\right\}$ induces a triangle in $W$, the centre $w$ of $W$ is either $v_{1}, v_{2}$ or $v_{3}$.

If $w=v_{2}$, then $v_{1}$ and $v_{3}$ are consecutive vertices on the rim of $W$. Then $G$ is the wheel obtained from $W$ by inserting the spoke $w x$. Hence $G \in \mathcal{T}_{2}$.

Now suppose $w=v_{1}$. Then we may suppose that $v_{3}$ is the successor of $v_{2}$ on the rim of $W$. Let $v_{3}^{+}$be the successor of $v_{3}$ on the rim of $W$. If $n(W) \geq 5$, then since $v_{1} v_{3} \notin E(G)$, it follows that $G\left[N\left(v_{3}^{+}\right)\right]$is not connected, contradicting that $G$ is LC. Hence $n(W)=4$, so $W \cong K_{4}$. This implies that $G$ is a wheel of order 5 , centred at $v_{2}$. Hence $G \in \mathcal{T}_{2}$.

Case 3. $m(G)=2 n(G)-1$ :

Suppose $G[N(x)]$ is a $K_{3}$ with vertex set $v_{1}, v_{2}, v_{3}$. Then $m(H)=2 n(H)-2$. Hence, by our induction hypothesis, $H$ is a wheel. Then $G\left[\left\{x, v_{1}, v_{2}, v_{3}\right\}\right]$ is a $K_{4}$, and $G$ is obtained by pasting this $K_{4}$ and the wheel $H$ together on the triangle $G\left[\left\{v_{1}, v_{2}, v_{3}\right\}\right]$. Thus $G$ is an extended $\theta$-graph, so $G \in \mathcal{T}_{1}$.

Now suppose $G[N(x)]$ is a path $v_{1} v_{2} v_{3}$. Then $m\left(H+v_{1} v_{3}\right)=2 n\left(H+v_{1} v_{3}\right)-1$, so by our induction hypothesis $H+v_{1} v_{3} \in \mathcal{T}_{1}$. There are three cases to consider.

(a) $H+v_{1} v_{3}$ is an extended $\theta$-graph: Let $u$ be the universal vertex of $H+v_{1} v_{3}$. Then $H+v_{1} v_{3}-u$ consists of two vertices $a$ and $z$ and three internally disjoint $a-z$ paths $P_{1}, P_{2}, P_{3}$. 


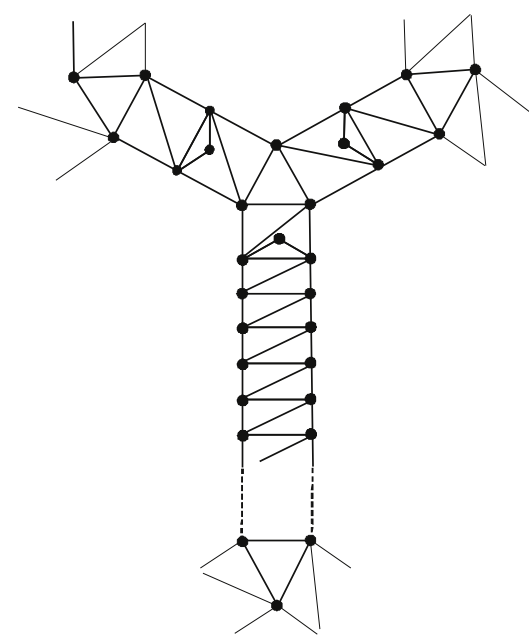

Fig. 10. Extending a border in $G^{*}$ to construct $G_{N}$.

If $u=v_{2}$, then $v_{1}$ and $v_{3}$ are two consecutive vertices on one of the paths $P_{1}, P_{2}, P_{3}$, and $G$ is obtained from $H+v_{1} v_{3}$ by inserting $x$ on the edge $v_{1} v_{3}$ and joining $x$ to $u$. So in this case $G$ is also an extended $\theta$-graph.

If $u=v_{1}$, then since $G\left[N\left(v_{3}\right)\right]$ is connected, we may assume that $P_{1}=a z$ and $P_{2}$ and $P_{3}$ have length 2 each. Let $y$ and $w$ be the internal vertices of $P_{2}$ and $P_{3}$, respectively. First, suppose $v_{2}=a$. Then $v_{2}$ is a universal vertex of $G$. If $v_{3}=z$, then $G-v_{2}$ consists of the three internally disjoint $v_{1}-v_{3}$ paths $v_{1} x v_{3}, v_{1} y v_{3}$ and $v_{1} w v_{3}$. If $v_{3}=y$, then $G-v_{2}$ consists of the three internally disjoint $v_{1}-z$ paths $v_{1} z, v_{1} x v_{3} z$ and $v_{1} w z$. In either case, $G$ is an extended $\theta$-graph. By symmetry, this proves all the cases when $v_{2} \in\{a, z\}$. If $v_{2} \notin\{a, z\}$, it is sufficient to consider the case $v_{2}=w, v_{3}=z$. Then $G$ consists of the wheels $G\left[\left\{v_{2}, a, v_{1}, x, v_{3}\right\}\right]$ and $G\left[\left\{a, v_{1}, v_{2}, v_{3}, y\right\}\right]$, pasted together on the diamond $G\left[\left\{a, v_{2}, v_{1}, v_{3}\right\}\right]$, so then $G$ is a diamond bicycle.

Now suppose $u \notin\left\{v_{1}, v_{2}, v_{3}\right\}$. Then we may assume that $P_{1}=a z$ and $P_{2}=a y z$, where $\{a, y, z\}=\left\{v_{1}, v_{2}, v_{3}\right\}$. If $y=v_{2}$, then $G$ consists of the wheel $G[\{y, u, a, x, z\}]$ and the wheel $G\left[\{u, y\} \cup V\left(P_{3}\right)\right]$, pasted together on the diamond $G[\{y, u, a, z\}]$. So in this case $G$ is a diamond bicycle. If $y=v_{1}$, then $G$ consists of the wheel $G[\{z, u, y, x, a\}]$ and the wheel $G\left[\{u\} \cup V\left(P_{3}\right)\right]$, pasted together on the triangle $G[\{u, z, a\}]$, so then $G$ is a triangle bicycle. In either case $G \in \mathcal{T}_{1}$.

(b) $H+v_{1} v_{3}$ is a triangle bicycle: Then $H+v_{1} v_{3}$ consists of two wheels $W$ and $Y$, each of order at least 5 , pasted together on a triangle $T$ such that $w$ and $y$, the respective centres of $W$ and $Y$, are distinct vertices in $T$. We may assume that $G\left[v_{1}, v_{2}, v_{3}\right]$ is a triangle in $W$. First, suppose $v_{1}$ or $v_{3}$ is the centre of $W$, say $v_{1}=w$. Then we may assume that $v_{3}$ is the successor of $v_{2}$ on the rim of $W$. Let $v_{3}^{+}$be the successor of $v_{3}$ on the rim of $W$. Now $v_{1} \notin N_{G}\left(v_{3}\right)$ and since $n(W) \geq 5$, there is no path from $v_{2}$ to $v_{3}^{+}$in $G\left[N\left(v_{3}\right)\right]$. Hence $G\left[N\left(v_{3}\right)\right]$ is not connected, contradicting that $G$ is LC. This proves that $\left\{v_{1}, v_{3}\right\} \cap\{w, y\}=\emptyset$ and hence $G\left[\left\{v_{1}, v_{2}, v_{3}\right\}\right] \neq T$.

We may therefore assume that $v_{2}=w$ and $v_{1} v_{3}$ is an edge on the rim of $W$. Since $y \notin\left\{v_{1}, v_{2}, v_{3}\right\}$, it follows that $v_{1} v_{3} \notin E(T)$. Now let $W^{*}$ be the wheel obtained from $W$ by subdividing the edge $v_{1} v_{3}$ with the vertex $x$ and adding the edge $x w$. Then $G$ is the triangle bicycle consisting of the wheels $W^{*}$ and $Y$, pasted together on the triangle $T$. Hence $G \in \mathcal{T}_{1}$.

(c) $H+v_{1} v_{3}$ is a diamond bicycle: Then $H+v_{1} v_{3}$ consists of two wheels $W$ and $Y$, each of order at least 5 , that share a diamond $D$ such that $w$ and $y$, the respective centres of $W$ and $Y$, are distinct vertices in $D$.

Suppose $v_{1}=w$. Then we may assume that $v_{3}$ is the successor of $v_{2}$ on $W$. Let $v_{3}^{+}$be the successor of $v_{3}$ on $W$. If $v_{3}=y$, then $G\left[N\left(v_{3}^{+}\right)\right]$is not connected, since there is no path from $v_{1}$ to $v_{3}$ in $N_{G}\left(v_{3}^{+}\right)$. If $v_{3} \neq y$, then there is no path from $v_{3}^{+}$to $v_{2}$, so then $G\left[N\left(v_{3}\right)\right]$ is not connected. This proves that $\left\{v_{1}, v_{3}\right\} \cap\{w, y\}=\emptyset$ and that $G\left[\left\{v_{1}, v_{2}, v_{3}\right\}\right]$ is not a triangle in $D$.

We may therefore assume that $v_{2}=w$ and $v_{1} v_{3}$ is an edge on the rim of $W$ such that $v_{1} v_{3}$ is not an edge of the diamond $D$. Hence $G$ is a diamond bicycle, consisting of two wheels pasted together on $D$.

Corollary 3.6. Every irreducible LC graph with $n \geq 3$ and $m \leq 2 n-1$ is hamiltonian.

\section{The hamilton cycle problem for sparse LC graphs}

A modification of the construction that produced the $\{2,5\}$-regular graph $G^{*}$ in the proof of Theorem 1.3 (see Fig. 3), yields the following result.

Theorem 4.1. For every natural number $k>1$ the Hamilton Cycle Problem for locally connected graphs with $m=2 n+\left\lfloor n^{1 / k}\right\rfloor$ is NP-complete. 
Proof. Consider any cubic graph $G$ of order $n$. Let $G^{*}$ be the $\{2,5\}$-regular graph obtained from $G$ as in Theorem 1.3. If $k>1$, we extend the border $B$ by successively attaching diamonds as shown in Fig. 10, until the resulting graph $G_{N}$ has $N=(3 n / 2)^{k}$ vertices. (Note that the order of $G^{*}$ is $3 n+(9 n / 2)$, which has the same parity as $3 n / 2$.)

This can be done in polynomial time. Each added vertex has degree 4 and the neighbours of vertices of degree 2 are of degree 5 in $G_{N}$. Hence the average degree of the vertices that are not in the node-set of $G_{N}$ is 4 . Note that $G_{N}$ has $n$ nodes and each node has three vertices, each of which has degree 5 . Hence

$$
\sum_{v \in V\left(G_{N}\right)} d(v)=5(3 n)+4(N-3 n)=4 N+3 n
$$

so

$$
\left|E\left(G_{N}\right)\right|=2 N+3 n / 2=2 N+N^{1 / k} .
$$

$G_{N}$ is clearly $L C$, and it follows easily from the proof of Theorem 1.3 that $G_{N}$ is hamiltonian if and only if $G$ is.

Our next theorem shows that if the value of $m$ in Theorem 4.1 is slightly decreased, the complexity of the problem is significantly reduced.

Theorem 4.2. For every natural number $k$ the Hamilton Cycle Problem for $L C$ graphs is in $\mathbb{P}$ for graphs with $2 n+k \log _{2} n$ edges or fewer.

The proof of Theorem 4.2 relies on the following reduction procedure, which decreases the order of an LC graph, while retaining local connectivity.

Reduction procedure. Let $G$ be an LC graph of order $n$ and size $2 n+p$, where $p$ is a positive integer. We choose a set of edges in $G$, which we refer to as special edges of $G$. We describe three types of reductions that may be performed on $G$.

Reduction 1: Suppose $v \in V(G)$ has degree 2, and let $N(v)=\{x, y\}$. Now delete the vertex $v$. The resulting graph $G^{\prime}$ is LC and $\left|E\left(G^{\prime}\right)\right|=2\left|V\left(G^{\prime}\right)\right|+p$. We observe that $G$ does not have a Hamilton cycle containing the edge $x y$. We also note that $G$ is hamiltonian if and only if $G^{\prime}$ has a Hamilton cycle containing the edge $x y$. We therefore add $x y$ to the set of special edges of the reduced graph.

Reduction 2: Suppose $G$ has a vertex $v$ of degree 3 such that $\langle N(v)\rangle$ is a path $x_{1} x_{2} x_{3}$. Then we delete $v$ and add the edge $x_{1} x_{3}$. The resulting graph $G^{\prime}$ is $L C$ and $\left|E\left(G^{\prime}\right)\right|=2\left|V\left(G^{\prime}\right)\right|+p$. We call $v$ a nice vertex of degree 3 . We note that $G^{\prime}$ is hamiltonian if $G$ is, but the converse need not be true.

Reduction 3: Suppose $G$ has a vertex $v$ of degree 3 such that $\langle N(v)\rangle$ is a triangle. Then we delete $v$. The resulting graph $G^{\prime}$ is LC and $\left|E\left(G^{\prime}\right)\right|=2\left|V\left(G^{\prime}\right)\right|+p-1$. We call $v$ a problematic vertex of degree 3. It follows from Proposition 3.1 that we meet at most $p+3$ problematic vertices of degree 3 when we successively delete vertices of degree 3 . We note that $G^{\prime}$ is hamiltonian if $G$ is, but the converse need not be true.

Next, we present some observations, lemmas and definitions that will be used to prove Theorem 4.2.

Observation 4.3. If we reduce a graph $G$ by removing a vertex $v$ by means of a Type 1,2 or 3 reduction, then the neighbours of $v$ are mutually adjacent in the resulting graph (even if two of the neighbours were not adjacent in $G$ ).

If $v$ is a vertex in a connected graph $G$ such that the neighbours of $v$ are mutually adjacent, then clearly $G-v$ is connected, so by Observation 4.3, Reductions 1, 2 and 3 do not create new vertex cuts. We shall apply this observation in the special version described in Lemma 4.4.

Lemma 4.4. Let $G$ be a connected $L C$ graph and let $F$ be a graph obtained from $G$ by means of a number of consecutive reductions of Type 1, 2 or 3. Let $z$ be the last vertex of $G$ that was removed to obtain $F$ and suppose $z$ had exactly two neighbours $x, y$ in $F$. Then $\{x, y\}$ is a vertex cut of $G$.

The square of an $n$-cycle, denoted by $C_{n}^{2}$, is the 4-regular graph obtained from the $n$-cycle $C_{n}$ by adding an edge between each pair of vertices at distance 2 from one another. The graph $C_{12}^{2}$ is depicted in Fig. 11.

Gordon et al. [4] proved the following.

Lemma 4.5. [[4]] Every connected 4-regular LC graph is the square of a cycle.

Let $v_{1} v_{2} \ldots v_{2 n} v_{1}$ denote the underlying $2 n$-cycle of the graph $C_{2 n}^{2}$. If the three edges between the sets $\left\{v_{1}, v_{2}\right\}$ and $\left\{v_{2 n-1}, v_{2 n}\right\}$ are deleted from $C_{2 n}^{2}$, the result is a zig-zag strip of order $2 n$. If the vertex $v_{2 n}$ is also deleted, the result is a zig-zag strip of order $2 n-1$. Thus a zig-zag strip consists of two "parallel" paths whose lengths differ by at most one, with edges between the paths in a zig-zag pattern. Fig. 12 depicts zig-zag strips of order 11 and 12.

Each triangle in a zig-zag strip may be viewed as a fan with two spokes, having its apex on one of the two parallel paths and its rim on the other. We call a graph obtained from a zig-zag strip by inserting spokes into such fans (as described in Section 3) a nice generalized strip. Note that any nice generalized strip is locally connected. Fig. 13 depicts an example of a nice generalized strip. 


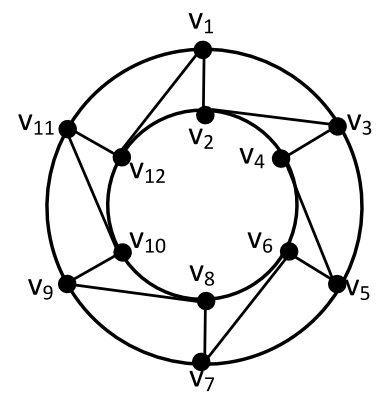

Fig. 11. The square of a 12-cycle.
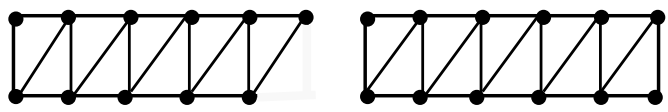

Fig. 12. Zig-zag strips of order 11 and 12.

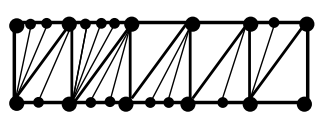

Fig. 13. An example of a nice generalized strip.

A fan in a graph $G$ is a nice fan if every internal vertex on the rim of the fan has degree 3 in $G$, i.e., only the end vertices of the rim and the centre may have neighbours outside the fan. We note that every fan in a nice generalized strip is a nice fan, provided its rim is on one of the two parallel paths.

A path cover of a graph is a set of vertex disjoint paths that contain all the vertices of the graph. An $r$-path cover is a path cover consisting of $r$ vertex disjoint paths.

Lemma 4.6. Let $S$ be a nice generalized strip and let $a_{1}, a_{2}$ and $b_{1}, b_{2}$ be the end vertices of the two parallel paths of S. Suppose $Q$ is a path cover of S such that the end vertices of all the paths in $Q$ are in $\left\{a_{1}, a_{2}, b_{1}, b_{2}\right\}$. Then there are at most 26 possibilities for the end vertices of the paths in $Q$.

Proof. If $Q$ is a 1-path cover (i.e., a Hamilton path), then the two end vertices can be chosen from the set $\left\{a_{1}, a_{2}, b_{1}, b_{2}\right\}$ in $\left(\begin{array}{l}4 \\ 2\end{array}\right)=6$ ways.

Now suppose $Q$ is a 2-path cover $\left\{Q_{1}, Q_{2}\right\}$. If neither $Q_{1}$ nor $Q_{2}$ is a singleton, then there are only 2 possibilities for their end vertices, since $S$ is planar. If $Q_{1}$ is a single vertex $v$, then there are 4 choices for $v$ and for each such choice there are 3 possibilities for the end-vertices of $Q_{2}$. Thus the total number of possibilities in the case of a 2-path cover is 14 .

If $Q$ is a 3-path cover, then two of the three paths in $Q$ are singletons, so in this case there are only 6 possibilities for the end vertices.

Thus the total number of possibilities is $6+14+6=26$.

If $G$ is a graph with a chosen set of special edges, we call a Hamilton cycle of $G$ that contains all the special edges of $G$ a special Hamilton cycle of $G$.

For simplicity, we only prove Theorem 4.2 for $k=1$, since it easily generalizes to an arbitrary natural number $k$. We actually prove the following slightly more general result for $k=1$.

Theorem 4.7. The problem of deciding whether an LC graph of order $n$ and size at most $2 n+\log _{2} n$ has a Hamilton cycle containing a set of prescribed edges is in $\mathbb{P}$.

We precede the proof with a brief outline, so that the reader can keep track of the main steps while going through the details of the proof.

Outline of proof. Let $G$ be an LC graph of order $n$ and size at most $2 n+\log _{2} n$, with a prescribed set of special edges. We use our reduction procedure to iteratively remove vertices of degree 2 and 3 , until we obtain a graph $H$ that has exactly $\left\lfloor\log _{2} n\right\rfloor$ vertices or has minimum degree 4, whichever occurs first. We choose the special edges of $H$ to consist of the special edges inherited from $G$, together with every edge that became special due to a Type 1 reduction. Then any special Hamilton cycle of $G$ reduces to a special Hamilton cycle of $H$, but the converse is not necessarily true.

We now reverse the reductions by first returning vertices of degree 3 as far as possible without returning vertices of degree 2, to obtain the graph $G^{*}$. When reversing a reduction, any edges that were added during the reduction are deleted, so $G^{*}$ is an induced subgraph of $G$. 


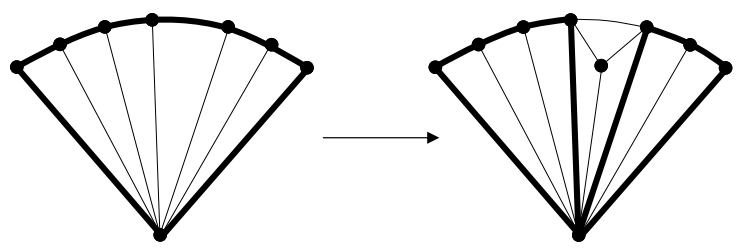

Fig. 14. Reversing a reduction of Type 3 divides a maximal nice fan into 2 maximal nice fans.

An analysis of the structure, order and size of $G^{*}$ allows us to shrink $G^{*}$ to a graph $H^{\prime}$ of sufficiently small size so that it can be checked in $O\left(n^{c}\right)$ time (for some constant $c$ ) whether $H^{\prime}$ has a special Hamilton cycle, and which is such that $H^{\prime}$ has a special Hamilton cycle if and only if $G^{*}$ has one.

If $G=G^{*}$, we are done. If not, there is at least one vertex $z$ in $G-V\left(G^{*}\right)$ that has two neighbours $x, y$ in $G^{*}$ and $x y$ is an edge that became special due to a Type 1 reduction. By Lemma 4.4, $\{x, y\}$ is a vertex cut of $G$, and since $G$ is locally connected, $G-\{x, y\}$ has exactly two components. Let $Z$ be the component of $G-\{x, y\}$ that contains $z$. Let $G_{x y}=G[V(Z) \cup\{x, y\}]$ and let $G_{1}=G\left[V\left(G^{*}\right) \cup V\left(G_{x y}\right)\right]$. Then $G_{1}$ has a special Hamilton cycle if and only if $G^{*}$ has a special Hamilton cycle and $G_{x y}$ has a special Hamilton cycle containing the edge $x y$. To check whether $G_{x y}$ has such a Hamilton cycle, we repeat our algorithm, starting with the 3-cycle $z x y z$ instead of the graph $H$. Again this is an $O\left(n^{c}\right)$ algorithm.

Since the number of Type 1 reductions that we need to reverse is less than $n$, repeated applications of the procedure result in an $O\left(n^{c+1}\right)$ algorithm for determining whether $G$ has a special Hamilton cycle.

Now we present the detailed proof.

Proof. Let $G$ be an LC graph of order $n$ and size at most $2 n+\log _{2} n$, with a given set of special edges. We may assume $G$ is connected, since otherwise the problem is trivial. We perform reductions 1,2 and 3 in any order until the resulting graph $H$ has precisely $\left\lfloor\log _{2} n\right\rfloor$ vertices or has minimum degree at least 4 . In either case it follows from the discussions of the reductions above that $|E(H)| \leq 2|V(H)|+\log _{2} n$.

Case 1. $H$ has precisely $\left\lfloor\log _{2} n\right\rfloor$ vertices:

In this case $|E(H)| \leq 2 \log _{2} n+\log _{2} n=3 \log _{2} n$. We consider each subset of $E(H)$ and check if that subset induces a special Hamilton cycle of $H$. This algorithm has time complexity $O\left(2^{|E(H)|} \log _{2} n\right)=O\left(2^{3 \log _{2} n} \log _{2} n\right)=O\left(n^{3} \log _{2} n\right)$.

We now focus on the maximal nice fans. We create the graph $G^{*}$ by reversing all the reductions of Types 2 and 3 that can be reversed without reversing any reductions of Type 1 . We note that $\left|E\left(G^{*}\right)\right| \leq 2\left|V\left(G^{*}\right)\right|+\log _{2} n$.

If a nice vertex whose neighbours form a triangle in a nice fan of order greater than 3 is returned by reversing a Type 2 reduction, then the edge of the triangle that is on the rim of the fan is deleted. (For, if a spoke were deleted we would not get an LC graph.) Thus, reversing a Type 2 reduction can extend a maximal nice fan but does not create additional maximal nice fans. Hence, if no Type 3 reductions were reversed to create $G^{*}$ from $H$, then the number of maximal nice fans in $G^{*}$ would be at most the number of edges of $H$, i.e., at most $3 \log _{2} n$.

However, we may need to return problematic vertices of degree 3 to create $G^{*}$. Returning a problematic vertex of degree 3 whose neighbours are a triangle in a maximal nice fan divides that fan into two edge disjoint maximal nice fans - see Fig. 14.

Since the number of problematic vertices that were deleted from $G$ to create $H$ is at $\operatorname{most}^{\log _{2}} n+3$ (as explained in the description of Reduction 3), the addition of problematic vertices to $H$ creates at most $\log _{2} n+3$ additional maximal nice fans. Thus the number of maximal nice fans in $G^{*}$ is at most $|E(H)|+\log _{2} n+3=4 \log _{2} n+3$.

We now reduce any maximal nice fan in $G^{*}$ that has more than 4 spokes to a nice fan with exactly four spokes, by eliminating internal spokes (as described in Section 3). Call the resulting graph $H^{\prime}$. Since eliminating a spoke amounts to reducing the number of vertices by one and the number of edges by two, it follows that $\left|E\left(H^{\prime}\right)\right| \leq 2\left|V\left(H^{\prime}\right)\right|+\log _{2} n$.

We may assume that the set of special edges in $G^{*}$ does not contain two non-consecutive internal spokes of any nice fan (since otherwise $G^{*}$, and hence $G$, will be nonhamiltonian). It is therefore possible to choose edges to be special in $H^{\prime}$ so that $H^{\prime}$ has a special Hamilton cycle if and only if $G^{*}$ has a special Hamilton cycle.

We note that if $F$ is a nice fan in $G^{*}$ that was obtained from a nice fan in $H$ by adding only nice vertices of degree 3 , the two outer spokes of $F$ are both in $H$. Thus, if $F$ is reduced to a fan with four spokes in $H^{\prime}$, it contains at most 2 vertices that were not in $H$.

If a problematic vertex of degree 3 is inserted into a triangle in a nice fan while we are building $G^{*}$, then two of the three the neighbours of that vertex lie on the outer spokes of two new maximal nice fans (see Fig. 14). Thus returning a problematic vertex results in at most 3 more vertices in $H^{\prime}$ that were not in $H$. Thus

$$
\left|V\left(H^{\prime}\right)\right| \leq|V(H)|+2\left(4 \log _{2} n+3\right)+3\left(\log _{2} n+3\right)=12 \log _{2} n+15
$$

and

$$
\left|E\left(H^{\prime}\right)\right| \leq 2\left|V\left(H^{\prime}\right)\right|+\log _{2} n \leq 25 \log _{2} n+30,
$$

so all the Hamilton cycles in $H^{\prime}$ can be found by means of an $O\left(2^{25 \log _{2} n+30} \log _{2} n\right)=O\left(n^{25} \log _{2} n\right)$ algorithm. 


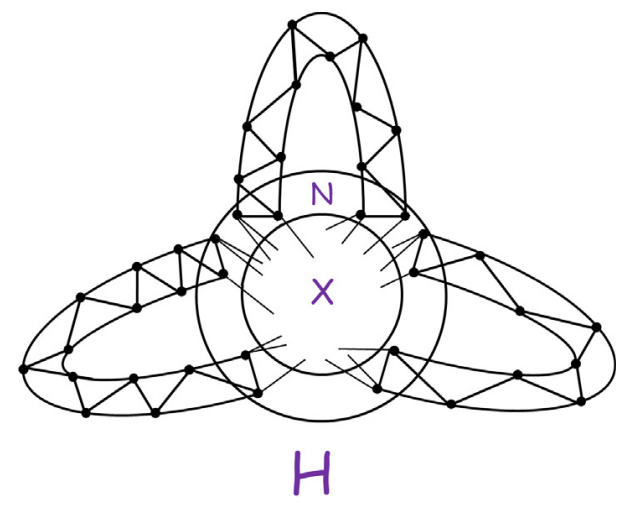

Fig. 15. Structure of $H$.

Thus, in this case there is an $O\left(n^{26}\right)$ algorithm for determining whether $G^{*}$ has a special Hamilton cycle.

If $G=G^{*}$, we are done. If not, we reverse a Type 1 reduction and carry on as explained in the last paragraph of the outline of the proof. The result is an $O\left(n^{27}\right)$ algorithm for determining whether $G$ has a special Hamilton cycle.

Case 2. $H$ has more than $\log _{2} n$ vertices (and $\delta(H) \geq 4$ ):

Recall that also in this case, $|E(H)| \leq 2|V(H)|+\log _{2} n$. Let

$$
X=\left\{v \in V(H): d_{H}(v)>4\right\}, N=N_{H-X}(X), M=G[X \cup N] .
$$

We assume that $X$ is nonempty. (Otherwise, the argument below is simpler.) Note that

$$
\sum_{v \in V(H)} d_{H}(v) \leq 4|V(H)|+2 \log _{2} n .
$$

Hence, since $\delta(H) \geq 4$, it follows that $\sum_{v \in X}\left(d_{H}(v)-4\right) \leq 2 \log _{2} n$, and hence $|X| \leq 2 \log _{2} n$. Now

$$
|N| \leq \sum_{v \in X} d_{H}(v)=\sum_{v \in V(H)} d_{H}(v)-\sum_{v \in V(H)-X} d_{H}(v) .
$$

But every vertex in $H-X$ has degree exactly 4 in $H$, so

$$
\begin{aligned}
|N| & \leq 4|V(H)|+2 \log _{2} n-4(|V(H)|-|X|) \\
& =4|X|+2 \log _{2} n \\
& \leq 10 \log _{2} n
\end{aligned}
$$

and

$$
|V(M)| \leq 12 \log _{2} n .
$$

Now

$$
\begin{aligned}
\sum_{v \in V(M)} d_{H}(v) & =4|V(M)|+\sum_{v \in X}\left(d_{H}(v)-4\right) \\
& \leq 4|V(M)|+2 \log _{2} n .
\end{aligned}
$$

Hence

$$
|E(M)| \leq 2|V(M)|+\log _{2} n \leq 25 \log _{2} n .
$$

Since every vertex in $H-X$ has exactly 4 neighbours in $H$, it can easily be proved using the same reasoning as in Lemma 4.5 that every component in $H-V(M)$ together with its neighbours in $N$ forms a zig-zag strip that has its two end edges in $N$ and all the other vertices in $H-V(M)-$ see Fig. 15.

Since every vertex in $N$ has degree 4 in $H$ and has at least one neighbour in $X$, no vertex in $N$ lies in more than one zig-zag strip of $H$, and each zig-zag strip in $H$ contains 4 vertices of $N$. Since $|N| \leq 10 \log _{2} n$, it follows that $H$ has at $\operatorname{most}_{5 / 2} \log _{2} n$ zig-zag strips.

We now create the graph $G^{*}$ by reversing all the reductions of Types 2 and 3 that can be reversed without reversing a reduction of Type 1 . We first return vertices that have three neighbours in a zig-zag strip. If only nice vertices are added to a zig-zag strip, that strip becomes a nice generalized strip. If a problematic vertex is added in a nice generalized strip, then the nice generalized strip becomes two nice generalized strips and we then add the problematic vertex and its neighbours to $M$, as illustrated in Fig. 16. Thus each addition of a problematic vertex to a nice generalized strip creates one more nice generalized strip and increases the number of vertices in $M$ by 4 and the number of edges by 6 . 

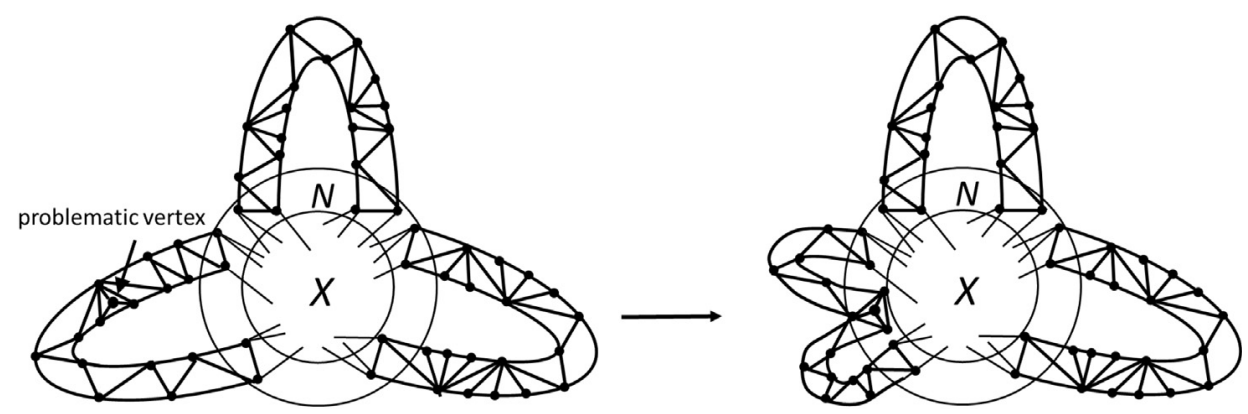

Fig. 16. Reversing reductions of Types 2 and 3 to create $G^{*}$.

Since there are at most $\log _{2} n+3$ problematic vertices, $|V(M)|$ increases to at most

$$
12 \log _{2} n+4\left(\log _{2} n+3\right)=16 \log _{2} n+12
$$

and $|E(M)|$ increases to at most

$$
25 \log _{2} n+6\left(\log _{2} n+3\right)=31 \log _{2} n+18,
$$

while the number of nice generalized strips increases to at most

$$
5 / 2 \log _{2} n+\log _{2} n+3=7 / 2 \log _{2} n+3 .
$$

The intersection of a Hamilton path of $G^{*}$ with each nice generalized strip is a path cover of which the end vertices of all the paths are in N. By Lemma 4.6 there are 26 possibilities for the end vertices of such path covers. For each of those, there is an $O(n)$ algorithm for deciding whether there exists a corresponding path cover containing all the special edges in the strip. Therefore, since there are at most $7 / 2 \log _{2} n+3$ nice generalized strips, there is an $O(n) \cdot 26^{7 / 2 \log _{2} n+3}<O(n) \cdot\left(2^{5}\right)^{7 / 2 \log _{2} n+3}<$ $O\left(n^{19}\right)$ algorithm for deciding whether there exist corresponding path covers containing all the special edges in all the strips.

So far we have returned vertices that have three neighbours in a zig-zag strip. We now return vertices whose neighbours induce triangles or paths with 3 vertices in $M$. Denote the resulting extension of $M$ by $M^{*}$. Using the method of Case 1 , we find that $M^{*}$ contains at most $|E(M)|+\log _{2} n+3=32 \log _{2} n+21$ maximal nice fans.

Now we reduce $M^{*}$ to the graph $M^{\prime}$ by reducing all the maximal nice fans in $M^{*}$ to fans with at most 4 spokes and choose appropriate special edges for $M^{\prime}$ as in Case 1. Then

$$
\begin{aligned}
& \left|V\left(M^{\prime}\right)\right| \leq|V(M)|+2\left(32 \log _{2} n+21\right)+3\left(\log _{2} n+3\right) \\
& =16 \log _{2} n+12+67 \log _{2} n+51=83 \log _{2} n+63,
\end{aligned}
$$

and

$$
\left|E\left(M^{\prime}\right)\right| \leq 2\left|V\left(M^{\prime}\right)\right|+\log _{2} n \leq 166 \log _{2} n+126 .
$$

Let $H^{\prime}$ denote the graph consisting of $M^{\prime}$ together with all the nice generalized strips. Then $H^{\prime}$ has a special Hamilton cycle if and only if $G^{*}$ has a special Hamilton cycle. For each subset of $E\left(M^{\prime}\right)$ and each choice of path covers of the strips we check, in $O(n)$ time, if the subsets of $E\left(M^{\prime}\right)$ and the path covers of the strips form a special Hamilton cycle of $H^{\prime}$. The complexity of this algorithm is at most $O\left(n^{19}\right) \cdot 2^{166 \log _{2} n+126} \cdot O(n)=O\left(n^{176}\right)$.

Thus, in this case there is an $O\left(n^{176}\right)$ algorithm for determining whether $G^{*}$ has a special Hamilton cycle.

If $G=G^{*}$, we are done. If not, we reverse a Type 1 reduction and carry on as explained in the last paragraph of the outline of the proof. The result is an $O\left(n^{177}\right)$ algorithm for determining whether $G$ has a special Hamilton cycle.

\section{Acknowledgements}

Thanks to the DST-NRF Centre of Excellence in Mathematical and Statistical Sciences (CoE-MaSS) for financial support. Opinions expressed and conclusions arrived at are those of the authors and are not necessarily to be attributed to the CoEMaSS.

First author was supported by the National Research Foundation of S.A., Grant number 81075. Second author was supported by the National Research Foundation of S.A., Grant number 103832. Third author was supported by the National Research Foundation of S.A., Grant number 107668. Fourth author was supported by ERC Advanced Grant GRACOL. 


\section{References}

[1] J.A. Bondy, U.S.R. Murty, Graph Theory, Springer, 2008.

[2] G. Chartrand, R.E. Pippert, Locally connected graphs, Časopis Pěst. Mat. 99 (1974) 158-163.

[3] L. Clark, Hamiltonian properties of connected locally connected graphs, Congr. Numer. 32 (1981) 199-204.

[4] V.S. Gordon, Y.L. Orlovich, C. Potts, V.A. Strusevich, Hamiltonian properties of locally connected graphs with bounded vertex degree, Discrete Appl. Math. 159 (2011) 1759-1774.

[5] G.R.T. Hendry, A strengthening of Kikust's theorem, J. Graph Theory 13 (1989) 257-260.

[6] G.R.T. Hendry, Extending cycles in graphs, Discrete Math. 85 (1990) 59-72.

[7] P.A. Irzhavski, Hamiltonicity of locally connected graphs: complexity results, Vestsi NAN Belarusi. Ser, Fiz-Mat. Navuk. 4 (2014) 3743 (in Russian). http://csl.bas-net.by/xfile/v_fizm/2014/4/6mrbl.pdf.

[8] P.B. Kikust, The existence of a hamiltonian cycle in a regular graph of degree 5 [Russian Latvian summary], Latvian Math. Yearbook 16 (1975) 33-38.

[9] C. Picouleau, Complexity of the hamiltonian cycle in regular graph problem, Theoret. Comput. Sci. 131 (1994) 463-473. 